\title{
LACTOBACILLUS BULGARICUS - THE CONTRIBUTION TO MODERN HEALTHY NUTRITION
}

\author{
Marieta Georgieva', Georgi Alexandrov ${ }^{2}$, Maria Peneva $^{3}$, Nikolai Manolov ${ }^{4}$ \\ ${ }^{1}$ Department of Pharmacology and Toxicology, Faculty of Pharmacy, Medical University \\ of Varna, ${ }^{2}$ Military Medical Academy of Sofia, ${ }^{3}$ MC Evrozdrave - Bulgaria, \\ ${ }^{4}$ Clinic of vascular surgery, Medical University of Varna
}

\begin{abstract}
Lactobacillus bulgaricus is part of the traditional Bulgarian food. It was known to the Thracians - the ancient population that lived on the territory of present Bulgaria.

At the beginning of $20^{\text {th }}$ century, the Russian scientist and Nobel prize winner Ilya Metchnikov, in his work „The Prolongation of Life: Optimistic Studies" relates the long and healthy life of Bulgarians to the yoghurt consumption and in particular to the local bacterium in yogurt.

In the $90 \mathrm{~s}$ of the $20^{\text {th }}$ century Lactobacillus bulgaricus was used in the production of probiotic functional food for astronauts. Using modern biotechnologies, certain Bulgarian companies sheathe strains of Lactobacillus bulgaricus and other probiotic microorganisms with a natural coating, consisting of components of the growing medium during fermentation. The isolation of new probiotic strains from spring water, which normally survive under the changing climatic conditions in nature, helps the production of probiotic products in which the strains of Lactobacillus bulgaricus and other probiotic microorganisms retain their stability and vitality when passing through the gastrointestinal tract.

Bulgaria becomes famous in the world for Lactobacillus bulgaricus and the Bulgarian territory is still an important reservoir for the isolation of natural strains of lactic acid bacteria which after an additional selection are used as starter cultures for the production of various fermented foods and probiotic products.

Lactobacillus bulgaricus is the ancient contribution of mankind to the modern agricultural science and the creation of the first healthy foods in the world.
\end{abstract}

Keywords: Lactobacillus bulgaricus, new probiotic strains, biotechnology, healthy foods

Lactobacillus bulgaricus is the only probiotic microorganism named after a certain territory and nation. It multiplies only on the territory of modern

\footnotetext{
Address for correspondence:

Marieta P. Georgieva, $M D, P h D$

Department of Pharmacology and Toxicology

Faculty of Pharmacy

Medical University of Varna

55 Marin Drinov Street, 9002 Varna, Bulgaria

e-mail:marieta_md@yahoo.com
}

Received: February 10, 2014

Accepted: April 3, 2014
Bulgaria - corresponding to ancient Thrace. In other regions of the world it mutates and stops its multiplication after 1-2 fermentations.

Lactobacillus bulgaricus is part of the Bulgarian traditional food. Bulgarians put Lactobacillus bulgaricus both in home and industry production of traditional lactic acid products - yoghurt, white brine cheese, other types of cheese and cream, milk-based infant formula, probiotic functional foods and whole food supplements (4).

Lactobacillus bulgaricus was known to the Thracians - the ancient population that lived in the present Bulgarian land more than $7-8$ thousand years 
ago. The word yoghurt is Thracian and means hard, solid milk. During his tour of Thrace the Greek scientist Herodotus $(484-425$ BC) wrote that the Thracians prepared special fermented dairy food which was a gift from their Gods (7).

In the archaeological excavations in 2007 Prof. Richard Evershed from the Bristol University found over 2200 ceramic vessels dated back to 9000 years in the region around the Black sea (present boundary of Bulgaria). Organic residues of fermented milk products were found in them.

In the beginning of $20^{\text {th }}$ century, the father of probiotics, the Russian scientist and a Nobel prize winner Ilya Metchnikov, in his work „The Prolongation of Life: Optimistics Studies" relates the long and healthy life of Bulgarians largely to the yoghurt consumption and in particular to the local bacterium in yoghurt (Fig. 1).

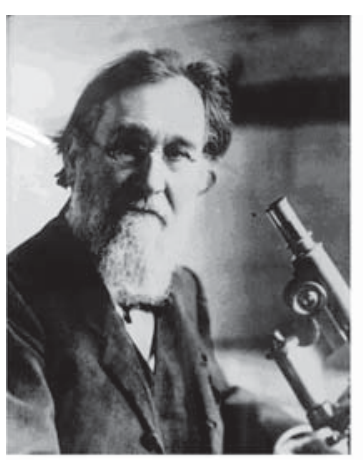

"Our aging

is a disease

that must be treated like any other desease."

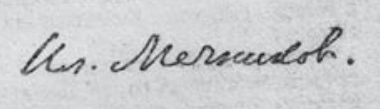

I. Mechnikov

1845-1916

Fig. 1. Prof. Illa Mechnikov

In 1905 in Geneva, the Bulgarian student St. Grigorov isolated Lactobacillus bulgaricus from yogurt, brought from Bulgaria (Fig. 2).

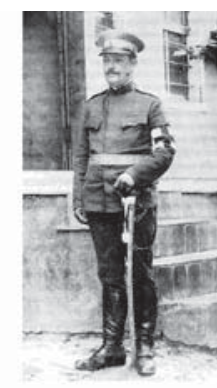

Stamen Grigorov Bulgarian Biologist and Physician

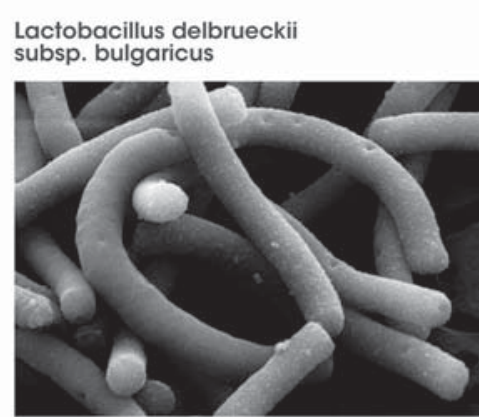

Fig. 2. Dr. Stamen Grigorov
Complying with the European standards of origin, a BSS (Bulgarian State Standard) for yoghurt was established in Bulgaria. According to this standard the lactic acid fermentation must be made only with Lactobacillus bulgaricus and Streptococcus thermophilus. According to the identity standards, published in the U.S. Code of Federal Laws, yoghurt is a fermented milk product that contains the typical bacterial cultures Lactobacillus bulgaricus and Streptococcus thermophilus.

In Commission Regulation (EU) No 432/2012 in the list of the permitted health claims made on foods, the only probiotic microorganisms included, are Lactobacillus bulgaricus and Streptococcus thermophilus. According to the claim, in doze over $10^{8}$ Colony forming units they improve lactose digestion (6).

In the 90 s of the $20^{\text {th }}$ century Lactobacillus bulgaricus was used in the production of probiotic functional food for astronauts that was tested during the flight of the second Bulgarian astronaut in Space (2).

Lactobacillus bulgaricus is of natural origin. It was considered until recently that it is of plant origin, i.e. it can be isolated from plants - cornel, rose calyx, etc. The Bulgarian scientists N. Alexandrov and D. Petrova isolated strains of Lactobacillus bulgaricus and other lactic acid probiotic microorganisms from spring water in Bulgaria (3).

Studies of many scientists from around the world prove the beneficial effects of Lactobacillus bulgaricus regarding regulation of functions and $\mathrm{mi}-$ crobial flora of the gastrointestinal tract, including diarrhea and disbacteriosis, immune system stimulation, reduction of the risk of cancer, regulation of the cholesterol level, competitive inhibition of pathogenic strains, causing infections, relief of lactose intolerance, antimutagenic and anti-radiation effect. Lactobacillus bulgaricus is closest to Döderlein flora in the woman's vagina and has a beneficial effect on the health of women and their newborns (1).

Lactobacillus bulgaricus causes homo-fermentative lactic acid fermentation in which it secretes only lactic acid and bacteriocins (beneficial substances), without alcohol and acetic acid production. During that fermentation Lactobacillus bulgaricus feeds on milk lactose, as the lactose remains in the lactic acid products in small quantities. In contrast, other probiotic bacteria induce hetero-fermentative lactic 
Marieta Georgieva, Georgi Alexandrov, Maria Peneva et al.

acid fermentation. Lactobacillus acidofilus and Lactobacillus lactis produce alcohol, and the Bifidobacteria produce acetic acid and small amounts of the beneficial lactic acid. Unlike other probiotic lactobacilli that secrete L (+) lactic acid, Lactobacillus bulgaricus secretes $\mathrm{L}(+)$ and $\mathrm{D}(-)$ lactic acid during lactic acid fermentation. It determines its powerful anti-cancer, ray-protective and antioxidant effects. Lactobacillus bulgaricus produces the antimicrobial substance Bulgarican, which is heat resistant and active against highly virulent (pathogenic) strains of microorganisms (5).

Studies of the Italian scientist S. Bianchi show that compared to the other lactic acid probiotic bacteria, Lactobacillus bulgaricus adheres best to the mucous membrane of the colon with subsequent rapid reproduction in the body. This determines its more powerful curative and detoxic effect as compared to the other lactic acid probiotic bacteria.

Lactobacillus bulgaricus reproduces best together with Streptococcus thermophilus. In a joint cultivation of Lactobacillus bulgaricus and Streptococcus thermophilus, there is a primary cooperation between them (associative link). It allows a fast accumulation of biomass and bioactive substances and potentiates their beneficial effects. In Bulgaria a lot of scientific studies of yeasts, containing Lactobacillus bulgaricus and Streptococcus thermophilus, used in yoghurt production were carried out. The results show that these specific bacteria of yoghurt are stable and survive when they pass through the human gastrointestinal tract.

Using modern biotechnology, certain Bulgarian companies sheathe strains of Lactobacillus bulgaricus and other probiotic microorganisms with a natural coating, consisting of components of the growing medium during fermentation. That and the isolation of new probiotic strains from spring water, which normally survive under the changing climatic conditions in nature, help the production of probiotic products in which the strains of Lactobacillus bulgaricus and other probiotic microorganisms retain their stability and vitality when passing through the gastrointestinal tract, as well as when stored in shops at a temperature up to $24^{\circ} \mathrm{C}$ for the entire shelf life.

Bulgaria becomes famous in the world for Lactobacillus bulgaricus and the Bulgarian territory is still an important reservoir for the isolation of natural strains of lactic acid bacteria which after an additional selection are used as starter cultures for the production of various fermented foods and probiotic products.

Lactobacillus bulgaricus is the ancient contribution of mankind to the modern agricultural science and to the creation of the first healthy foods in the world.

\section{REFERENCES}

1. Alexandrov N, Petrova D, Kovacheva M. The probiotics - base of functional nutrition and nutritive treatment in XXI century. In: International Symposium on original Bulgarian yoghurt, Sofia; 2005. p. 125-6.

2. Alexandrov N. Prophylactic and treatment of multiple organ failure in poisoning with halogenated hydrocarbons. In: Symp of the Army Medical Services of Member States of Warsaw treaty. National Defense Ministry; 1988. p. 17.

3. Application for Patent No. 13557597/25.07.2012 in the U.S. Patent Office and Application No. 111161/07.03.2012 in Bulgarian Patent Office, Probiotics for dietary diary product.

4. Chomakov H. Bulgarian Yoghurt - unique probiotic International Symposium on original Bulgarian yoghurt, Sofia; 2005. p. 21-35.

5. Chomakov H., Probiotics. Sofia; 2007.

6. Commission Regulation (EU) No 432/2012 .

7. Hosono A. Nutritive and physiological properties of lactic acid bacteria. Nagano, Japan: Japanese International Cooperation Agency (JICA), Agricultural faculty, Shinshu University; 1996. p. 30-2. 